\title{
In-orbit calibration of the lunar magnetometer onboard SELENE (KAGUYA)
}

\author{
Futoshi Takahashi ${ }^{1}$, Hisayoshi Shimizu ${ }^{2}$, Masaki Matsushima ${ }^{1}$, Hidetoshi Shibuya ${ }^{3}$, Ayako Matsuoka ${ }^{4}$, \\ Satoru Nakazawa ${ }^{5}$, Yuichi Iijima ${ }^{4}$, Hisashi Otake ${ }^{5}$, and Hideo Tsunakawa ${ }^{1}$ \\ ${ }^{1}$ Department of Earth and Planetary Sciences, Tokyo Institute of Technology, Tokyo 152-8551, Japan \\ ${ }^{2}$ Earthquake Research Institute, University of Tokyo, Tokyo 113-0032, Japan \\ ${ }^{3}$ Department of Earth and Environmental Sciences, Kumamoto University, Kumamoto 860-8555, Japan \\ ${ }^{4}$ Institute of Space and Astronautical Science, Japan Aerospace Exploration Agency, Sagamihara, Kanagawa 229-8510, Japan \\ ${ }^{5}$ Lunar and Planetary Exploration Program Group, Japan Aerospace Exploration Agency, Tsukuba, Ibaraki 305-8505, Japan
}

(Received September 3, 2008; Revised June 2, 2009; Accepted August 2, 2009; Online published December 21, 2009)

\begin{abstract}
The high-sensitivity fluxgate Lunar MAGnetometer (LMAG) is mounted on SELENE (KAGUYA) to investigate the near-surface electromagnetic environment and the evolution of the Moon through magnetic field observation. To avoid possible electromagnetic interferences, a triaxial fluxgate sensor (MGF-S) is installed at the far end of a 12-m-long mast. It is critical for the accurate observation to monitor MGF-S alignment in orbit, and thus we have calibrated the sensor alignment by measuring the known magnetic fields generated by the sensor alignment monitor coil (SAM-C) wound onto the mast canister. In-orbit calibration of the MGF-S alignment was performed twice each revolution during the initial check-out phase of the satellite. It is concluded that there is no systematic difference in the sensor alignment between the day-side and night-side. Applying a new technique based on the Davis-Smith method to the observed magnetic field data when KAGUYA was exposed to the solar wind, a zero offset of each axis was quickly and stably determined every month. As a result, LMAG has been calibrated with an accuracy that is sufficient for detection of the lunar magnetic anomaly at an altitude of $100 \mathrm{~km}$ and for high-resolution electron reflectometry.
\end{abstract}

Key words: SELENE (KAGUYA), lunar magnetic field, magnetometer, in-orbit calibration.

\section{Introduction}

The SELENE (KAGUYA) spacecraft launched on September 14, 2007 has been maintaining a near polar orbit of about a $100-\mathrm{km}$ altitude as designed. The Lunar MAGnetometer (LMAG) is mounted on KAGUYA to measure the magnetic fields originating from the Moon, such as the magnetic anomalies on the surface (e.g., Hood et al., 1979, 2001; Kurata et al., 2005; Toyoshima et al., 2008), and time-dependent magnetic responses containing information on the electrical conductivity of the Moon's interior (e.g., Hobbs, 1973; Dyal et al., 1976; Russell et al., 1981; Hood et al., 1999) as well as the interplanetary magnetic field. The field intensity of the lunar crustal magnetic anomalies and the induction response are estimated to be $1 \mathrm{nT}$ or less at this altitude. The spatial resolution of the electron reflection (ER) method is planned to be comparable to the cyclotron radius of low-energy electrons, i.e., about $5 \mathrm{~km}$ (Halekas et al., 2001). For these measurements, two in-orbit calibrations are of particular importance: determination of the sensor alignment and estimation of zero offsets. To make the systematic error less than $0.08 \mathrm{nT}$ when the magnetic field is observed in the Earth's magnetosphere ( $\sim 10 \mathrm{nT})$, the sensor alignment must be determined with an accuracy of better than $0.5^{\circ}$. Mapping the surface intensity by the ER method, the observed point is determined by extrapola-

Copyright (c) The Society of Geomagnetism and Earth, Planetary and Space Sciences (SGEPSS); The Seismological Society of Japan; The Volcanological Society of Japan; The Geodetic Society of Japan; The Japanese Society for Planetary Sciences; TERRAPUB tion of the in-situ magnetic field onto the lunar surface. If the zero offset is estimated with an accuracy of better than $0.5 \mathrm{nT}$, the accuracy of the in-situ field direction is about $3^{\circ}$ in the magnetosphere $(\sim 10 \mathrm{nT})$ and then the spatial resolution of the ER method at a nominal altitude of $100 \mathrm{~km}$ is about $5 \mathrm{~km}$ on the lunar surface.

The LMAG has a triaxial ring-core type fluxgate sensor (MGF-S), designed to be a highly linear instrument with a low noise level, at the tip of the 12-m long mast to avoid possible electromagnetic interference. All of the scientific instruments and bus devices were designed and improved to satisfy the strict electromagnetic compatibility (EMC) condition of the magnetic field: a magnetic field interference $<0.02 \mathrm{nT}$ in DC and $0.006 \mathrm{nT}$ in $\mathrm{AC}<32 \mathrm{~Hz}$ at the position of the MGF-S for the respective instruments. The ground tests confirmed that the specification of the MGF-S summarized in Table 1 is satisfied (Shimizu et al., 2008), and that the LMAG can observe the lunar magnetic field under a magnetically clean environment (Nakazawa et al., 2008). However, it was difficult to check the magnetometer in the ground test deploying the mast under the same temperature and gravity conditions as those in orbit.

The sensor alignment can be calibrated in orbit by observing the geomagnetic main field when the satellite is orbiting the Earth (Olsen et al., 2003) or passing nearby the Earth for swingby (Anderson et al., 2001, 2007; Dougherty et al., 2004; Glassmeier et al., 2007). In the case of LMAG, the mast is $12 \mathrm{~m}$ long and its temperature variation with KAGUYA's revolution is conceivably comparable to the lu- 
Table 1. Summary of specification of the magnetometer and ground calibration result.

\begin{tabular}{|c|c|c|}
\hline Range number & Dynamic range & Resolution (16 bit) \\
\hline 0 & $\pm 64 \mathrm{nT}$ & $0.002 \mathrm{nT}$ \\
\hline 1 & $\pm 256 \mathrm{nT}$ & $0.008 \mathrm{nT}$ \\
\hline 2 & $\pm 1024 \mathrm{nT}$ & $0.03 \mathrm{nT}$ \\
\hline 3 & $\pm 65536 \mathrm{nT}$ & $2.0 \mathrm{nT}$ \\
\hline \multicolumn{2}{|c|}{ Linearity } & Better than $10^{-3}$ \\
\hline \multicolumn{2}{|c|}{ Noise level } & $<0.1 \mathrm{nT}$ \\
\hline \multicolumn{2}{|c|}{ Sampling rate } & $32 \mathrm{~Hz}$ \\
\hline \multicolumn{2}{|c|}{ Orthogonality } & Within $0.4^{\circ}$ \\
\hline \multicolumn{2}{|c|}{ Sensitivity } & Within $0.2 \%$ error \\
\hline \multirow[t]{2}{*}{ Temperature dependence } & Sensitivity & Stable within $0.3 \%$ between $-45^{\circ} \mathrm{C}$ to $32^{\circ} \mathrm{C}$ \\
\hline & Offset & Stable between $-35^{\circ} \mathrm{C}$ to $33^{\circ} \mathrm{C}$ \\
\hline
\end{tabular}

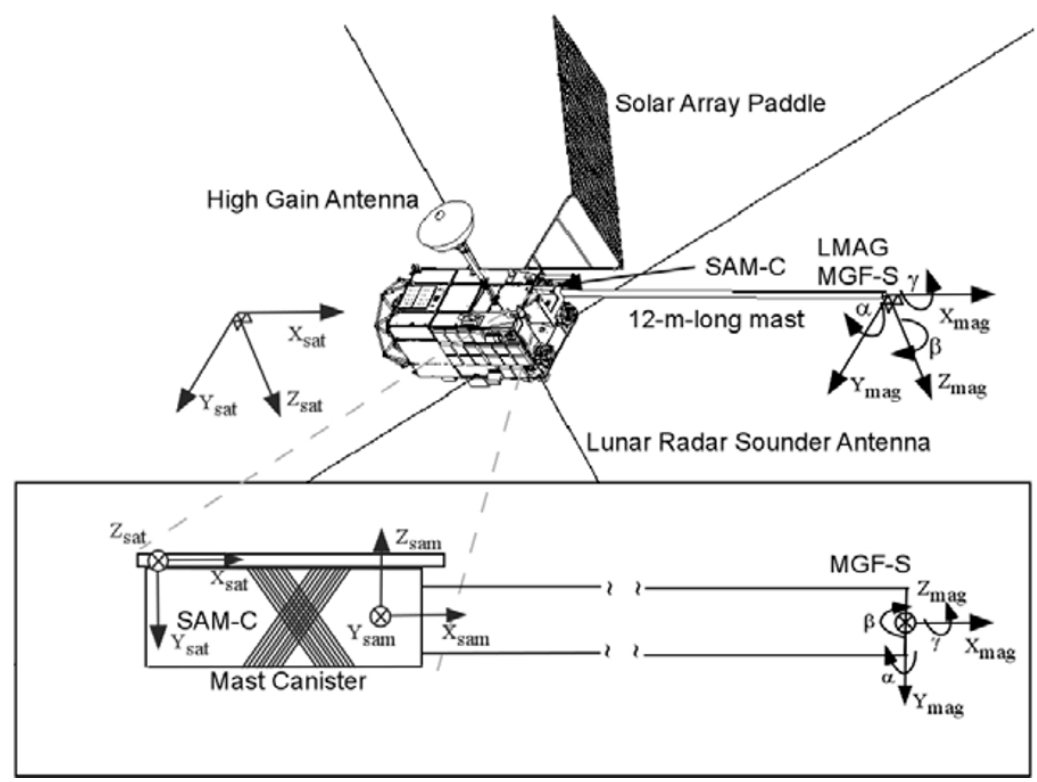

Fig. 1. Schematic view of the KAGUYA spacecraft showing the relationship among the magnetometer (mag), SAM-C (sam), and satellite (sat) coordinate frames with the Euler angles $(\alpha, \beta, \gamma)$. Indicated is the mast canister and SAM-C in close-up view seen from the $-Z_{\mathrm{sat}}$ direction.

nar diurnal change at the surface $\left(-170\right.$ to $\left.110^{\circ} \mathrm{C}\right)$. In order to make a precise observation, we have included the capability to monitor for possible deformation of the mast due to temperature change. KAGUYA has the sensor alignment monitor system with two coils (SAM-C), that generate an artificial magnetic field and which are wound onto the mast canister stowed in the spacecraft (Fig. 1; Shimizu et al., 2008). The SAM system is similar to the Cassini's in-flight calibration system called the science calibration subsystem (SCAS; Dougherty et al., 2004). In this paper, we show results of the in-orbit calibration of the LMAG, the sensor alignment monitored by the SAM-C, and the estimated zero offsets.

\section{Methods of In-orbit Calibration}

As shown in Table 1, orthogonality and linearity of the LMAG were confirmed in the ground calibration experiment (Shimizu et al., 2008). Also, no clear temperature dependencies of the offset, sensitivity, and SAM-C were found. Therefore, we do not explicitly assume temperature dependence of the LMAG in the following analyses, although its validity is to be checked as a result.

\subsection{Linear inversion problem using the SAM-C system}

The SAM-C consists of bi-axis coils, SAM-C(A) and SAM-C(B), that generate linearly independent magnetic fields with a $1-\mathrm{Hz}$ triangular wave form of about $2.6 \mathrm{nT}$ amplitude at the sensor position for $10 \mathrm{~s}$ and gradually decrease the amplitude in $8 \mathrm{~s}$. Shimizu et al. (2008) estimate that alignment angles (Euler angles in Fig. 1) can be determined with a precision of $0.1^{\circ}$ based on a large number of measurements in the calibration with a $32-\mathrm{Hz}$ sampling frequency.

The sensor alignment is analyzed in the sensor coordinate system using the SAM-C current data monitored simultaneously. The raw magnetic field data generally include a SAM-C generating field, an external field, various noises, and an instrumental offset. In addition, we found that a small bias of a 1-Hz rectangular wave form is superimposed and synchronized with increasing and decreasing SAM-C current. This bias is believed to be caused by an electromotive force due to small loop circuits of the connector at the bottom of 12-m mast very near the SAM-C. This will be discussed later. In order to extract the SAM-C generating field efficiently, we assume that the observed field in the 
calibration at the time $t_{n}(n=1, \cdots, N)$ is composed of a trend component, a triangular component responding to SAM-C current, a rectangular bias responding to increasing and decreasing SAM-C current, and random noise as

$$
B_{i n}^{\mathrm{OBS}}=B_{i n}^{\mathrm{TRE}}+J_{n}^{\mathrm{SAM}} f_{i}^{\mathrm{RES}}-s_{n}^{J} f_{i}^{\mathrm{BIA}}+B_{i n}^{\mathrm{RND}},
$$

where $B_{i n}^{\mathrm{OBS}}$ is the $i$-th component of the raw magnetic field data $(i=x, y, z)$ at $t=t_{n}, B_{i n}^{\mathrm{TRE}}$ is the $i$-th trend component, $J_{n}^{\mathrm{SAM}}$ is a SAM-C current, $f_{i}^{\mathrm{RES}}$ is the $i$-th response factor, $f_{i}^{\mathrm{BIA}}$ is the $i$-th bias factor, $B_{i n}^{\mathrm{RND}}$ is random noise, and $s_{n}^{J}$ is a coefficient depending on temporal change of the SAM-C current, $\Delta J_{n}^{\text {SAM: }} s_{n}^{J}=1$ for $\Delta J_{n}^{\text {SAM }}>0$ and $s_{n}^{J}=-1$ for $\Delta J_{n}^{\mathrm{SAM}}<0$. In the above equation, a constant instrumental offset and smooth change in the external field are included in the trend component, while random noise contains the external field fluctuation, the instrumental noise and, possibly, the interference field, which is negligibly small at the sensor position.

We apply the Bayesian statistical procedure using a quadratic $B$-spline function with $(M-1)$ knots (Tsunakawa, $1992,1995)$ to carry out the decomposition. Physically, it is assumed for this procedure that spline coefficients vary smoothly. Also, the trade-off parameter, $\lambda>0$, should be introduced to represent the smoothness. In Bayesian statistics, we assume that the random noise and second difference of the spline coefficients with respect to time are independently subject to normal distributions with zero mean and variances, $\sigma_{1}$ and $\sigma_{2}$, respectively, and that the ratio of the variances is $\lambda=\sigma_{1} / \sigma_{2}$ (Tsunakawa, 1992). The likelihood function $l(y \mid x)$ of unknown parameters $(x)$ from the data $(y)$ distribution and the prior distribution $\pi(x)$ are related to the posteriori distribution $\pi^{\text {post }}(x)$ by Bayes theorem: $\pi^{\text {post }}(x) \propto l(y \mid x) \pi(x)$. In this case,

$$
\begin{aligned}
\pi_{i}^{\text {post }} & =\left(\sqrt{\frac{1}{2 \pi \sigma_{1}^{2}}}\right)^{n}\left(\sqrt{\frac{\lambda}{2 \pi \sigma_{1}^{2}}}\right)^{m} \exp \left(-\frac{S_{i}}{2 \sigma_{1}^{2}}\right), \\
S_{i}= & \sum_{n=1}^{N}\left\{B_{i n}^{\mathrm{OBS}}-\left(B_{i n}^{\mathrm{TRE}}+J_{n}^{\mathrm{SAM}} f_{i}^{\mathrm{RES}}-s_{n}^{J} f_{i}^{\mathrm{BIA}}\right)\right\}^{2} \\
& +\lambda \sum_{n=1}^{m}\left(\Delta^{2} B_{i n}^{\mathrm{TRE}}\right)^{2},
\end{aligned}
$$

where $q=b_{n}^{2}-c^{2}$, which should be constant $(=q)$ if $b_{n}$ is

posteriori distribution for a given prior distribution, which cannot be determined until the optimal value of $\lambda$ is obtained. Such $\lambda$ can be found by minimization of ABIC (Akaike's Bayesian Information Criterion: Akaike, 1980). In the present case,

$$
\begin{aligned}
\mathrm{ABIC} & \equiv-2 \ln \int \pi_{\text {post }}(x) d x \\
& =N \ln \frac{S_{i}^{* 2}}{N}+\ln \left[\operatorname{det}\left(X^{\mathrm{T}} X\right)\right]-M \ln \lambda+\text { const },
\end{aligned}
$$

where $N$ is the number of the observed data points, $S_{i}^{*}$ is a temporary value of $S_{i}$ with a given $\lambda$, and $X$ is the $(N+$ $M) \times M$ matrix consisting of the quadratic spline functions and the damping matrix (Tsunakawa, 1992). Once $\lambda$ is constant, that is, Alfvénic. The above equation is expressed as

$$
U_{1} \mathbf{x}=\mathbf{z}
$$

where

$$
U_{1}=\left(\begin{array}{cccc}
2 B_{x 1} & 2 B_{y 1} & 2 B_{z 1} & 1 \\
2 B_{x 2} & 2 B_{y 2} & 2 B_{z 2} & 1 \\
\vdots & \vdots & \vdots & \vdots \\
2 B_{x N} & 2 B_{y N} & 2 B_{z N} & 1
\end{array}\right)
$$

determined by utilizing ABIC, $B_{i n}^{\mathrm{TRE}}, f_{i}^{\mathrm{RES}}, f_{i}^{\mathrm{BIA}}$, and $B_{i n}^{\mathrm{RND}}$ are obtained by minimizing $S_{i}$. The rotation angles are then calculated from $f_{i}^{\text {RES }}$ using the ground test results (Shimizu al., 2008).

Since KAGUYA is a three-axis stabilized spacecraft revolving the Moon, the Davis-Smith (D-S) method (Davis and Smith, 1968; Belcher, 1973) is applicable to the offdetermination of three axes. The D-S method is based constant regardless of direction when the Alfvén wave is observed. Defining the time series of the observed field, the Alfvén wave and the offset by $\mathbf{B}_{n}=\left(B_{x n}, B_{y n}, B_{z n}\right)$, $\mathbf{b}_{n}=\left(b_{x n}, b_{y n}, b_{z n}\right)$, and $\mathbf{c}=\left(c_{x}, c_{y}, c_{z}\right)$ at the time $t_{n}$, ectively, we obtain the following equations,

$$
\begin{aligned}
& b_{x n}^{2}+b_{y n}^{2}+b_{z n}^{2}=b_{n}^{2}, \\
& c_{x}=\text { const, } c_{y}=\text { const, } c_{z}=\text { const, }|\mathbf{c}|=\operatorname{const}(=c) .
\end{aligned}
$$

The D-S method supposes the offset $\mathbf{c}$ to minimize the variance of $b_{n}^{2}$, and the following equation is obtained,

$U_{0} \mathbf{c}=\left(\left\langle B^{2} \mathbf{B}\right\rangle-\left\langle B^{2}\right\rangle\langle\mathbf{B}\rangle\right) / 2$,

where mean values are represented by \langle\rangle . In the present analysis, we adopt another equation derived as follows. The observed data consist of $\mathbf{b}_{n}$ and $\mathbf{c}$,

$$
\mathbf{B}_{n}=\mathbf{b}_{n}+\mathbf{c} .
$$

Therefore, we have

$$
2 B_{x n} c_{x}+2 B_{y n} c_{y}+2 B_{z n} c_{z}+q=B_{n}^{2},
$$

and

$$
\begin{aligned}
& \mathbf{x}=\left(c_{x}, c_{y}, c_{z}, q\right)^{\mathrm{T}}, \\
& \mathbf{z}=\left(B_{1}^{2}, B_{2}^{2}, \cdots, B_{N}^{2}\right)^{\mathrm{T}} .
\end{aligned}
$$

The unknown vector $\mathbf{x}$ is determined by the least square fitting method. This equation is mathematically equivalent to the original D-S equation (proof is given in Appendix A). Since this alternative form of the D-S equation is numerically less sensitive to the round-off error and, therefore, more stable for a large number of the observed data points, 

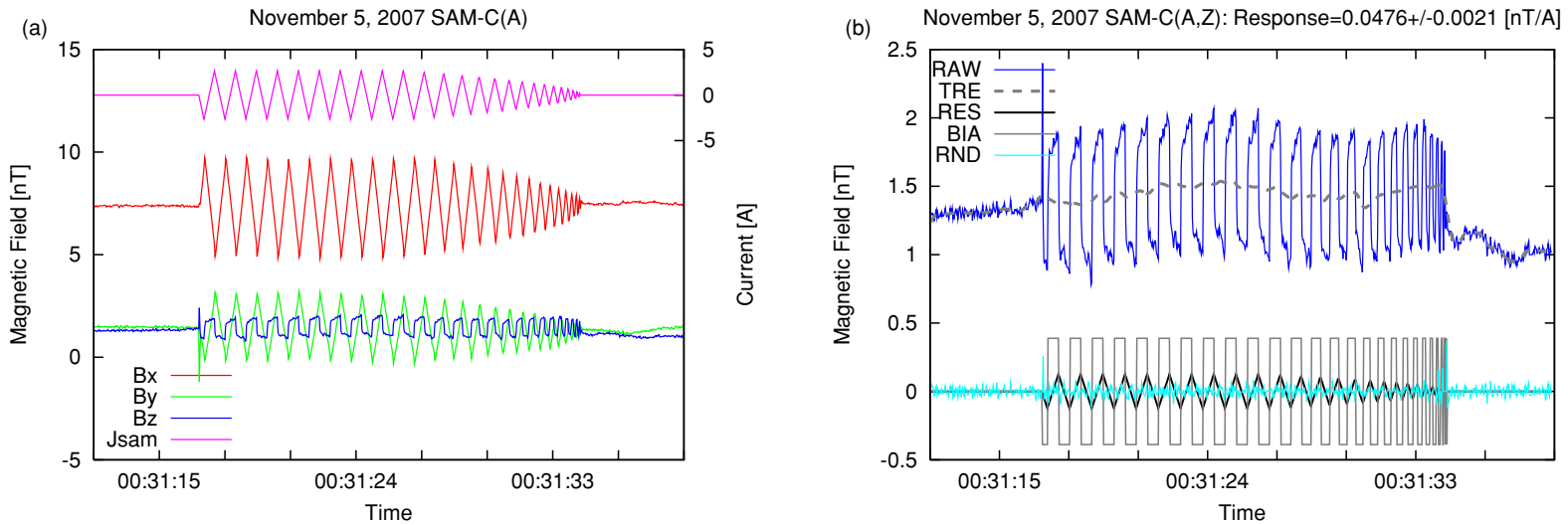

Fig. 2. (a) Time series of the magnetic field at 32-Hz sampling frequency and driving electric current to the SAM-C during the quiet magnetic environment on November 5, 2007. (b) Results of the time-series analysis in quiet ambient magnetic field for the $z$ component. The external trend (gray dashed line), response (black solid line), bias (gray solid line), and random noise (cyan) components are shown as well as the raw data (blue). $x, y$, and $z$ are in the magnetometer coordinate system.

we solve Eq. (11) to determine the offsets instead of Eq. (7). This new technique of the D-S method is applied to 1-s average data of 10 -min segment $(N=600)$ in the sensor coordinate system during November 2007-March 2008 when KAGUYA was exposed to the solar wind. Using the obtained offsets, 1-day and 1-month means are calculated. Since the LMAG should automatically select the range- 0 $( \pm 64 \mathrm{nT}$, Table 1$)$ in the orbit of $100-\mathrm{km}$ altitude, we determine the offsets for the range- 0 .

\section{Results of In-orbit Calibration}

Calibration of the MGF-S alignment using the SAM-C was carried out once each on the day-side and night-side of the Moon during the initial check-out phase of KAGUYA scientific operations in order to examine if significant deformation of the mast may occur due to severe temperature change. Figure 2(a) shows an example of the observed magnetic field in 1/32-s resolution, together with the driving electric current for SAM-C, $J_{n}^{\text {SAM }}$, during the calibration made on November 5, 2007, in the solar wind. As mentioned previously, bias of a rectangular wave form is superimposed on the SAM-C generating field of a triangular wave form, especially in the $z$ component. Therefore, the bias term is taken into account in the processing method.

Figure 2(b) shows the observed magnetic field separated into a trend, a response with a triangular wave form, a bias with a rectangular wave form, and random components. The response factor, $f_{i}^{\mathrm{RES}}$, which is equivalent to a ratio of the SAM-C generating field to $J_{n}^{\text {SAM }}$, has been thus determined and used to obtain the Euler angles of the MGF-S.

We occasionally saw large deviations of the response factor from the average level. When such a deviated factor appeared, in most cases magnetic disturbances with relatively high spectra at $1 \mathrm{~Hz}$ contaminated the SAM-C generating field. Such inappropriate data can be distinguished and rejected if the Fourier power spectrum of the observed magnetic field does not have dominant power at $1 \mathrm{~Hz}$. Table 2 summarizes the Euler angles of $(\alpha, \beta, \gamma)$ in the day-side and night-side during November 5-15, 2007, which are determined at a precision of about $0.1^{\circ}$. In this period, temperatures of the sensor range from $-0.7^{\circ} \mathrm{C}$ to $15^{\circ} \mathrm{C}$. The
Table 2. Euler angles of the MGF-S determined by in-orbit calibrations. Estimation errors are denoted by the standard error. The unit is degree.

\begin{tabular}{lccc}
\hline & $\alpha$ & $\beta$ & $\gamma$ \\
\hline C/O phase day-side & $-0.05 \pm 0.03$ & $-0.78 \pm 0.07$ & $-4.16 \pm 0.08$ \\
C/O phase night-side & $-0.03 \pm 0.02$ & $-0.87 \pm 0.04$ & $-4.09 \pm 0.07$ \\
\hline
\end{tabular}

Table 3. The monthly averaged offset values. Estimation errors are denoted by the standard error. The unit is nT.

\begin{tabular}{lcrc}
\hline & $c_{x}$ & $c_{y}$ & $c_{z}$ \\
\hline November 2007 & $3.23 \pm 0.24$ & $-0.53 \pm 0.31$ & $-1.41 \pm 0.22$ \\
December 2007 & $3.19 \pm 0.25$ & $1.25 \pm 0.44$ & $-1.73 \pm 0.28$ \\
January 2008 & $2.95 \pm 0.32$ & $1.74 \pm 0.62$ & $-1.56 \pm 0.23$ \\
February 2008 & $3.56 \pm 0.15$ & $1.13 \pm 0.65$ & $-1.32 \pm 0.09$ \\
March 2008 & $3.37 \pm 0.15$ & $1.20 \pm 0.43$ & $-1.28 \pm 0.16$ \\
\hline
\end{tabular}

difference in the rotation angles between the day-side and night-side is less than $0.1^{\circ}$, and thus we may assume that sensor alignment does not vary between the day-side and night-side.

The monthly means of the offsets are shown in Fig. 3 and Table 3. Both of the $x$ and $z$ offsets are well determined with standard errors of $0.1-0.6 \mathrm{nT}$ and are stable through this period. The $y$ offset has relatively larger errors of monthly average values and seems to have changed by about 2 nT after November 2007. The cause of the $y$-offset change may conceivably be an aging effect, as since this time it has been almost the same. Therefore, all of three offsets can be regarded as being stable within $\pm 0.3 \mathrm{nT}$ since December, 2007.

Subtracting the offsets, applying the Euler's rotation angles, and subsequently multiplying matrices for the coordinate transformation, we finally have the calibrated magnetic field in the systems, such as the GSE (Geocentric Solar Ecliptic), SSE (Selenocentric Solar Ecliptic), and ME (Mean Earth/Polar Axis) coordinate systems. In order to check validity of the in-orbit calibrations, the time series of the magnetic field observed by KAGUYA/LMAG is compared with that of ACE on January 28, 2008 (Fig. 4), as was done for the NEAR (Lohr et al., 1997) and ROSETTA 

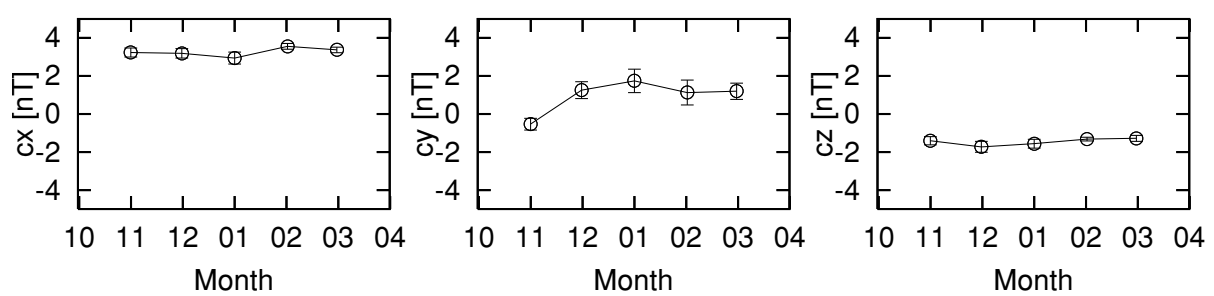

Fig. 3. The monthly averaged offset values between November 2007 and March 2008. Error bars represent the standard error.

January 28, 2008 in GSE

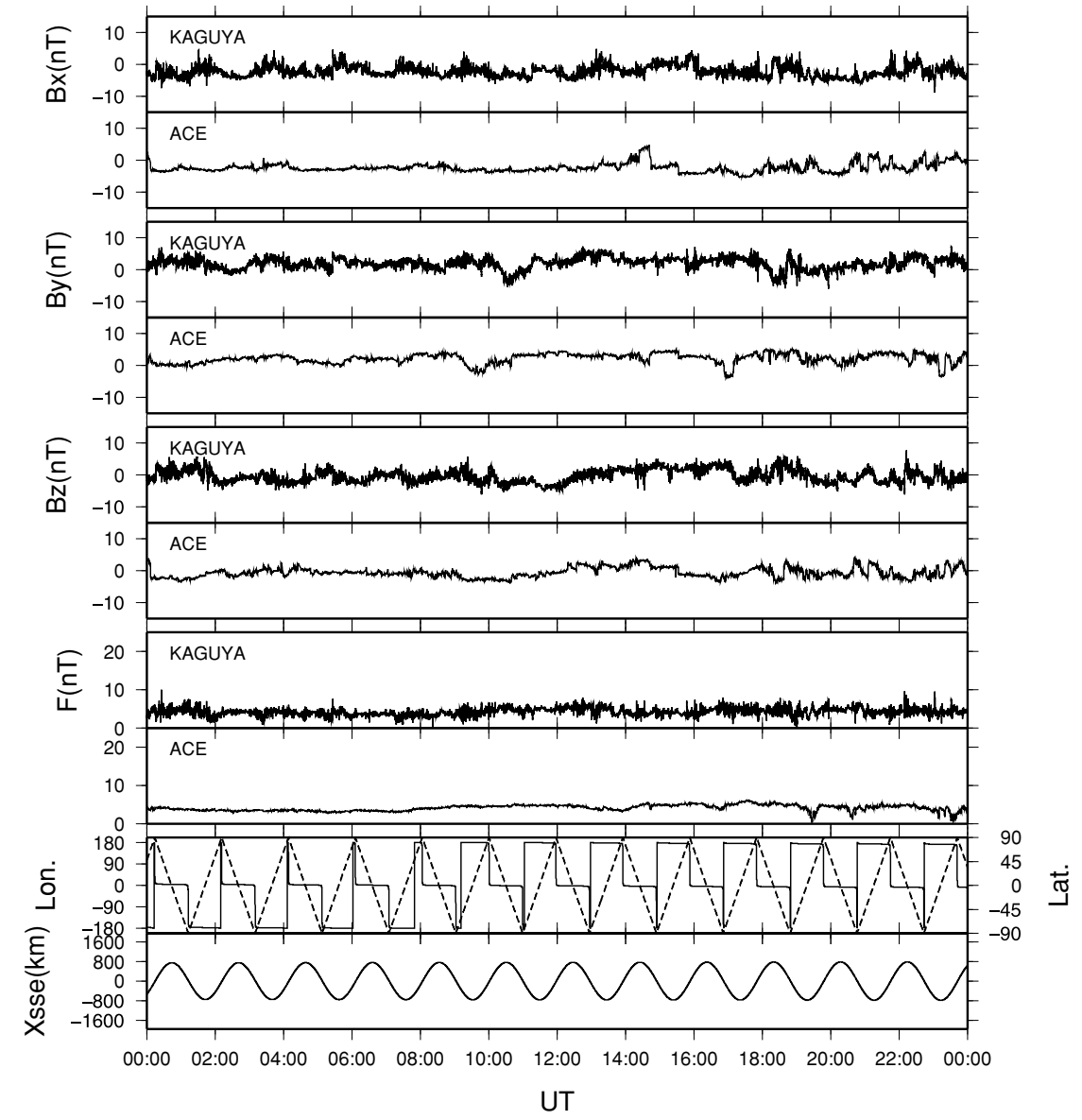

Fig. 4. Time series of the magnetic field ( $B_{x}, B_{y}, B_{z}$, and $F$ (total force)) on January 28,2008 observed by KAGUYA/LMAG are compared with the ACE data. The LMAG data and ACE level-2 data obtained at http://cdaweb.gsfc.nasa.gov/ are 1-s and 16-s values, respectively, in the Geocentric Solar Ecliptic (GSE) coordinate system. KAGUYA's positions are also shown in the body-fixed selenographic latitude (dashed line) and longitude (solid line) and in the X component of the Selenocentric Solar Ecliptic (SSE) coordinate system.

(Glassmeier et al., 2007) with the WIND spacecraft. Taking account of about a 1-h delay due to the distance between the two spacecrafts $\left(250-270 R_{\mathrm{E}}\right.$, where $R_{\mathrm{E}}$ is the Earth's radius), the magnetic field observed by KAGUYA is very similar to that by ACE except for some modifications due to the solar wind interaction at the terminator and the wake region on the night-side. Hence, we have confirmed that the sensor alignment and zero offsets are well determined in the present analysis.

\section{Concluding Remarks}

In-orbit calibration of the KAGUYA lunar magnetometer, LMAG, was primarily carried out during the initial check- out phase. The sensor alignment is almost the same on the day-side and night-side of the Moon within $\pm 0.1^{\circ}$. However, we should also check the secular change in alignment, and it will be assessed when we have data covering a sufficiently long time span. Therefore, alignment calibration was carried out twice a month to monitor a possible slow alignment change. The zero offsets have been almost stable within $\pm 0.3 \mathrm{nT}$ since December, 2007. The present results basically meet the requirement for LMAG scientific objectives. Further improvement in offset determination may be required to clearly detect the induction signature of the Moon. To this end, an advanced algorithm by Leinweber et al. (2008) could be useful, as was done for the Venus 
Express magnetometer (Zhang et al., 2008). Comparison of the magnetic field data obtained by KAGUYA and ACE indicates that the in-orbit calibrations have been well performed and therefore enabled us to detect the lunar magnetic anomaly field of about $1 \mathrm{nT}$ even at the altitude of $100 \mathrm{~km}$ and to measure the lunar surface intensity by the ER method with a high spatial resolution of about $5 \mathrm{~km}$.

Acknowledgments. The authors wish to express their sincere thanks to all of the SELENE team member, especially to M. Kato, K. Tanaka, S. Sobue, H. Hoshino, H. Okumura, Y. Yamamoto, J. Kimura, and H. Maejima, for various help in the observation. We are also grateful to T. Omoto at Meisei Electric Co., and Y. Takeuchi at NIPPI Co. for great support in developing the instrument. Helpful comments by two anonymous referees are greatly appreciated.

\section{Appendix A.}

Shown here is a brief proof that the modified D-S equation (11) used in this study is equivalent to the original one (Eq. (7)). Taking the mean of Eq. (10) gives

$$
2\left\langle B_{x}\right\rangle c_{x}+2\left\langle B_{y}\right\rangle c_{y}+2\left\langle B_{z}\right\rangle c_{z}+q=\left\langle B^{2}\right\rangle .
$$

Then, subtracting Eq. (A.1) from Eq. (10), we obtain

$$
\begin{aligned}
& 2\left(B_{x n}-\left\langle B_{x}\right\rangle\right) c_{x}+2\left(B_{y n}-\left\langle B_{y}\right\rangle\right) c_{y}+2\left(B_{z n}-\left\langle B_{z}\right\rangle\right) c_{z} \\
& \quad=B_{n}^{2}-\left\langle B^{2}\right\rangle .
\end{aligned}
$$

Using a matrix $U_{2}$ and a vector $\mathbf{z}_{2}$, we have $U_{2} \mathbf{c}=\mathbf{z}_{2} / 2$, where

$$
\begin{gathered}
U_{2}=\left(\begin{array}{ccc}
B_{x 1}-\left\langle B_{x}\right\rangle & B_{y 1}-\left\langle B_{y}\right\rangle & B_{z 1}-\left\langle B_{z}\right\rangle \\
B_{x 2}-\left\langle B_{x}\right\rangle & B_{y 2}-\left\langle B_{y}\right\rangle & B_{z 2}-\left\langle B_{z}\right\rangle \\
\vdots & \vdots & \vdots \\
B_{x N}-\left\langle B_{x}\right\rangle & B_{y N}-\left\langle B_{y}\right\rangle & B_{z N}-\left\langle B_{z}\right\rangle
\end{array}\right), \\
\mathbf{z}_{2}=\left(B_{1}^{2}-\left\langle B^{2}\right\rangle, B_{2}^{2}-\left\langle B^{2}\right\rangle, \cdots, B_{N}^{2}-\left\langle B^{2}\right\rangle\right)^{\mathrm{T}} .
\end{gathered}
$$

Multiplying the transpose, $U_{2}^{\mathrm{T}}$ and division by $N$ yield

$$
\frac{1}{N} U_{2}^{\mathrm{T}} U_{2} \mathbf{c}=\frac{1}{2 N} U_{2}^{\mathrm{T}} \mathbf{z}_{2} .
$$

This equation is identical with Eq. (7). For example,

$$
\begin{aligned}
\frac{1}{N}\left(U_{2}^{\mathrm{T}} U_{2}\right)_{1,1} & =\frac{1}{N} \sum_{i=1}^{N}\left(B_{x n}-\left\langle B_{x}\right\rangle\right)^{2} \\
& =\left\langle B_{x}^{2}\right\rangle-\left\langle B_{x}\right\rangle^{2} \\
& =\left(U_{0}\right)_{1,1} .
\end{aligned}
$$

\section{References}

Akaike, H., Likelihood and the Bayes procedure, in Bayesian Statistics, edited by Bernardo, J. M., M. H. DeGroot, D. V. Lindley, and A. F. M. Smith, pp. 143-166, University Press, Valencia, Spain, 1980.

Anderson, B. J., L. J. Zanetti, D. H. Lohr, J. R. Hayes, M. H. Acuña, C. T. Russell, and T. Mulligan, In-flight calibration of the NEAR magnetometer, IEEE Trans. Geosci. Remote Sens., 39, 907-917, 2001.

Anderson, B. J., M. H. Acuña, D. H. Lohr, J. Scheifele, A. Raval, H. Korth, and J. A. Slavin, The magnetometer instrument on MESSENGER, Space Sci. Rev., 131, 417-450, 2007.

Belcher, J. W., A variation of the Davis-Smith method for in-flight determination of spacecraft magnetic fields, J. Geophys. Res., 78, 6480, 1973.

Davis, L. Jr. and E. J. Smith, The in-flight determination of spacecraft magnetic field zeros, Trans. AGU, 49, 257, 1968.
Dougherty, M. K., S. Kellock, D. J. Southwood, A. Balogh, E. J. Smith, B. T. Tsurutani, B. Gerlach, K.-H. Glassmeier, F. Gleim, C. T. Russell, G. Erdos, F. M. Neubauer, and S. W. H. Cowley, The Cassini magnetic field investigation, Space Sci. Rev., 114, 331-383, 2004.

Dyal, P., C. W. Parkin, and W. D. Daily, Structure of the lunar interior from magnetic field measurements, Proc. Lunar Sci. Conf. 7th, 3077-3095, 1976.

Glassmeier, K.-H., I. Richter, A. Diedrich, G. Musmann, U. Auster, U. Motschmann, A. Balogh, C. Carr, E. Cupido, A. Coates, M. Rother, K. Schwingenschuh, K. Szegö, and B. Tsurutani, RPC-MAG The fluxgate magnetometer in the ROSETTA Plasma Consortium, Space Sci. Rev. 128, 649-670, 2007.

Halekas, J. S., D. L. Mitchell, R. P. Lin, S. Frey, L. L. Hood, M. H. Acuña and A. B. Binder, Mapping of crustal magnetic anomalies on the lunar near side by the Lunar Prospector electron reflectometer, J. Geophys. Res., 106, 27841-27852, 2001.

Hobbs, B. A., The inverse problem of the moon's electrical conductivity, Earth Planet. Sci. Lett., 17, 380-384, 1973.

Hood, L. L., P. J. Coleman, Jr., and D. E. Wilhelms, The moon: sources of the crustal magnetic anomalies, Science, 204, 53-57, 1979.

Hood, L. L., D. L. Mitchell, R. P. Lin, M. H. Acuña, and A. B. Binder, Initial measurements of lunar induced magnetic dipole moment using Lunar Prospector magnetometer, Geophys. Res. Lett., 26, 2327-2330, 1999.

Hood, L. L., A. Zakharian, J. Halekas, D. L. Mitchell, R. P. Lin, M. H. Acuña, and A. B. Binder, Initial mapping and interpretation of lunar crustal magnetic anomalies using Lunar Prospector magnetometer data, J. Geophys. Res., 106, 27825-27839, 2001.

Kurata, M., H. Tsunakawa, Y. Saito, H. Shibuya, M. Matsushima, and H. Shimizu, Mini-magnetosphere over the Reiner Gamma magnetic anomaly region on the moon, Geophys. Res. Lett., 32, L24205, doi:10.1029/2005GL024097, 2005.

Leinweber, H. K., C. T. Russell, K. Torkar, T. L. Zhang, and V. Angelopoulos, An advanced approach to finding magnetometer zero levels in the interplanetary magnetic field, Meas. Sci. Technol., 19, 055104, doi:10.1088/0957-0233/19/5/055104, 2008.

Lohr, D. A., L. J. Zanetti, B. J. Anderson, T. A. Potemra, J. R. Hayes, R. E. Gold, R. M. Henshaw, F. F. Mobrey, and D. B. Holland, NEAR magnetic field investigation, instrumentation, spacecraft magnetic and data access, Space Sci. Rev., 82, 255-281, 1997.

Nakazawa, S., Y. Iijima, H. Tsunakawa, M. Matsushima, T. Ono, A. Kumamoto, Y. Kasahara, S. Ikegami, and T. Ishikawa, Electromagnetic compatibility of SELENE (KAGUYA), 26th International Symposium on Space Technology and Science, 2008-k-24, 2008.

Olsen, N., L. Toeffiner-Clausen, T. J. Sabaka, P. Brauer, J. M. G. Merayo, J. L. Joergensen, J.-M. Léger, O. V. Nielsen, F. Primdahl, and T. Risbo, Calibration of the Oersted vector magnetometer, Earth Planets Space, 55, 11-18, 2003.

Russell, C. T., P. J. Coleman, Jr., and B. E. Goldstein, Measurements of the lunar induced magnetic moment in the geomagnetic tail: Evidence for a lunar core?, Proc. Lunar Planet. Sci. Conf. 12th, 831-836, 1981.

Shimizu, H., F. Takahashi, N. Horii, A. Matsuoka, M. Matsushima, H. Shibuya, and H. Tsunakawa, Ground calibration of the high-sensitivity SELENE lunar magnetometer LMAG, Earth Planets Space, 60, 353363, 2008.

Toyoshima, M., H. Shibuya, M. Matsushima, H. Shimizu, and H. Tsunakawa, Equivalent source mapping of the lunar crustal magnetic field using ABIC, Earth Planets Space, 60, 365-373, 2008.

Tsunakawa, H., Bayesian approach to smoothing palaeomagnetic data using ABIC, Geophys. J. Int., 108, 801-811, 1992.

Tsunakawa, H., Deconvolution method for directions of the postdepositional detrital remanent magnetization using an exponential fixing function, J. Geomag. Geoelectr., 47, 551-576, 1995.

Zhang, T. L., M. Delva, W. Baumjohann, M. Volwerk, C. T. Russell, S. Barabash, M. Balikhin, S. Pope, K.-H. Glassmeier, K. Kudela, C. Wang, Z. Vörös, and W. Zambelli, Initial Venus Express magnetic field observations of the Venus bow shock location at solar minimum, Planet. Space Sci., 56, 785-789, 2008.

F. Takahashi (e-mail: futoshi@geo.titech.ac.jp), H. Shimizu, M. Matsushima, H. Shibuya, A. Matsuoka, S. Nakazawa, Y. Iijima, H. Otake, and $\mathrm{H}$. Tsunakawa 